\title{
Features of formation of karst falls on the earth's surface
}

\author{
Vitaly Trofimov ${ }^{1}$ and Ivan Shipovskii ${ }^{1, *}$ \\ ${ }^{1}$ Institute of Comprehensive Exploitation of Mineral Resources, Russian Academy of Sciences, \\ 111020, Moscow, Russia
}

\begin{abstract}
Karst manifestations are widespread in many regions and pose a significant danger to residence and economic activity. Failing funnels arise during the collapse of rocks over underground voids (caves, workings, etc.), formed during karst formation or in the process of anthropogenic doing in the rock massive. However, not every karst or technogenic cavity gives rise to a failure of the earth's surface, and as a rule, its occurrence is unexpected. In this work, we consider the dynamics of the formation of dips of the earth's surface in the form of a collapse pipe. To do this, the geomechanical problem is solved by the meshless code Smoothed Particle Hydrodynamics (SPH). The method allows to obtain a solution to the problem taking into account large deformations and possible discontinuities in the process of changing the stress-strain state. The Drucker-Prager fracture criterion is used, the parameters of which change over time in accordance with the accumulation of damage, which determines the temporary development of the fracture process, its beginning and speed. Various options for the formation of a vertical dip are considered depending on the geometrical parameters of the initial cavity, its depth and materials composing the rock mass, as well as the features of the destruction of various materials composing the mass during the formation of the dip. Relations are obtained that relate the depth of the cavity, the horizontal size of the hole, the strength properties of the rocks (adhesion, angle of internal friction), the coefficient of lateral pressure in the array. The features of wave processes generated by the formation of a dip are considered, for which a velocity field is obtained near the fracture zone at various time points in the fracture process. Key words: karst failure, computer simulation, Drucker-Prager fracture criterion, meshless SPH code.
\end{abstract}

\section{Introduction}

Significant surface areas of the Earth's land are formed by karst rocks, which can pose a great danger of the occurrence of failures of the earth's surface and its subsidence in the form of small, but often appearing craters (fig. 1) [1].

\footnotetext{
*Corresponding author: shipovskiy_i@ipkonran.ru
} 


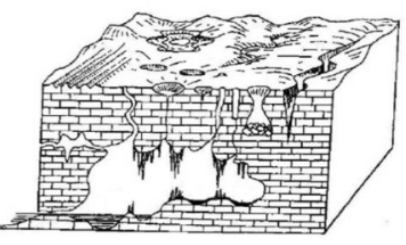

Fig.1. Karst landforms.

Karst is a complex geological process caused by the dissolution of underground and (or) surface waters of rocks, manifested in their weakening, destruction [2, 3].

In the modern classification, 4 types of karst are distinguished: open, covered, overlapped and overlapped-covered, depending on the presence of the overburden and the properties of its rocks [4]. The interest of geological engineers, environmentalists, designers and builders in this problem is certainly justified, since such manifestations can disrupt the normal operation of ground and underground structures and threaten people's lives.

The karst manifestations, with the exception of open ones, at the first stages have no visible signs of manifestation and are associated with the formation and development of zones of softening and softening in the overburden. Over time, they transform into cavities and caves, possibly filled with water. After this, the overburden may collapse with the formation of a funnel.

Today, there are various geophysical methods for predicting karst occurrences at the stage of formation and development of softening and softening zones. In this case, the formation of a funnel is only a matter of time and is mainly determined by the deformationstrength properties of the rocks. A characteristic feature of such funnels in many cases is the presence at the early stage of vertical walls, which in time become flattened due to rock shedding. Sometimes such walls do not form and subsidence is formed in the form of a smooth trough.

By objects and research methods, the problem under consideration can be divided into two parts. The first is to identify weakened areas, determine their shape, size and degree of filling with bulk material and water. The main role in obtaining this data belongs to field research methods: engineering-geological, hydrogeochemical, geophysical, speleological, etc. When solving some issues, it is advisable to use the methods of physical and mathematical modeling.

The second part of the problem is the study of the destruction process itself, the patterns of deformation and destruction of the soil of the covering layer, the mechanisms of funnels formation. The solution to this particular problem is the focus of this article.

\section{Theory}

If the stresses around the karst cavity do not reach critical values, then based on the calculation of the stress-strain state of the array near the cavity in the framework of the stationary model, it is impossible to predict its evolution and assess the risk of dynamic destruction of the surrounding rock mass.

In the framework of that model, a rock mass with a karst cavity does not collapse, and cracks and surface damage can develop only under additional load, for example, during the construction of a massive object near the cavity [4]. In practice, the destruction of the surface can occur in the absence of a significant anthropogenic power load and often not immediately, but after a certain, sometimes very large, time interval. Modeling karst using classical strength criteria may be inadequate, since the actual development of cracks in the massif often begins and occurs at stresses below a critical value. This is mainly due to the fact that over time the geometric parameters of the cavity change due to its growth and a redistribution of the stress-strain state of the rock mass occurs due to this increase. In 
parallel with this, the process of rock destruction and accumulation of damage in them, i.e. degradation deformation-strength properties of the rock.

In the proposed numerical model, the process is considered to occur in time on the basis of solving the complete system of equations of mechanics of a deformable solid, together with the damage accumulation equation. The strength parameters of the medium deteriorate in accordance with the value of the destruction $D$.

The damage accumulation equation used in this article, as well as the elastic-plastic model, are presented in [7, 8]. Earlier, using this approach, a solution to a number of practical problems was demonstrated in [7-9].

In addition, the model uses the plastic model Drucker-Prager. In this case, the elastic state of the medium in the stress space is limited by the surface of the limiting state. Exceeding the values of the environmental parameters of these boundaries starts the process of inelastic deformation in accordance with the equations of the limiting surface and plasticity potential.

$$
f\left(\sigma_{i j}, \varepsilon_{i j}^{p}\right)=0 \text {, }
$$

where $f$ is the equation of the loading surface. Plastic deformation is determined from the equation

$$
d \varepsilon_{i j}^{p}=d \lambda \frac{\partial g}{\partial \sigma_{i j}},
$$

where $g\left(\sigma_{i j}, \varepsilon_{i j}^{p}\right)=0$ is the plastic potential; $d \lambda$ finding out in the process of deformation from the condition of plasticity; $\varepsilon_{\mathrm{ij}}^{\mathrm{p}}$ - components of the plastic strain tensor.

The limiting surface in the region of shear strain in the pressure range $P^{*} \leq P \leq P_{0}$ is described by the equation

and at pressures $P>P_{0}$ - by equation

$$
f_{1}(P, \tau)=\tau-\alpha P-Y
$$

$$
f_{2}(P, \tau)=\frac{\left(P-P_{0}\right)^{2}}{a^{2}}+\frac{\tau^{2}}{b^{2}}-1=0 .
$$

Here $\tau=\left(\frac{1}{2} S_{i j} S_{i j}\right)^{1 / 2}$ is the shear stress intensity; $\mathrm{P}^{*}$ is the pressure at which brittle fracture occurs by separation; $P_{0}$ is the threshold pressure at which compaction of the material begins; $a=\left(P_{1}-P_{0}\right), b=c+\alpha P_{0}$.

The parameters $\alpha=\alpha\left(e^{p}, P\right), Y=Y\left(e^{p}, P\right)$, which describe the Drucker-Prager surface, can be expressed in terms of the angle of internal friction $\varphi$ and cohesion $\mathrm{c}$ of the Coulomb-Mohr model.

The change in the strength of the loaded medium over time can be taken into account by introducing the function of damage accumulation

$$
D\left(\sigma_{i j}, t\right)=\int \frac{\left(S-S_{0}\right)^{n}}{\left(S^{*}\right)^{n} t^{*}} d t, \text { for } S>S_{0},
$$

where $S$ is the effective stress; $S_{0}, S^{*}, t^{*}, n$ - parameters that determine the threshold stress, starting from which damage is accumulated, ultimate stress and parameters characterizing the rate of damage accumulation. In this case, the equation describing the hardening and softening is supplemented by the factor $\left(1-D\left(\sigma_{i j}, t\right)\right)$, containing the kinetic function (5).

With this approach of continuum fracture mechanics, the relations of the theory of plasticity are often used, and the separation into plastic and brittle deformation becomes conditional. In this case, the volumetric changes associated with the formation of microcracks are taken into account without going to the macro level and, accordingly, without using special procedures for describing cracks. This approach is justified in the case of multiple fracture without the formation of main cracks. Its application is especially effective in problems associated with the deformation of rocks under compression and shear. 
Thus, damage is considered as an increase in a non-decreasing variable $D$ that corresponds to the current stress state and the state of damage. This is interpreted as the beginning of crack formation. Damage changes some properties of the material. Under dynamic conditions, this enables the spatial localization of areas of rapidly increasing damage, accompanied by loosening, which changes the picture of the stress-strain state of the surrounding rock. An important property of damage parameters is their interaction and feedback with other parameters of the stress - strain state.

It is believed that the weakening, softening and disengagement, as a result of damage, are functionally set through the modules:

$$
K=\left(1-D^{2}\right) K_{0}, \mu=\left(1-D^{2}\right) \mu_{0}, \tau=\left(1-D^{2}\right) \tau_{0},
$$

Wherein softening the material from damage caused by stretching reduces the bulk modulus $K$, the shear modulus $\mu$, and the yield strength $\tau$ from their basic values for intact material $\mathrm{K}_{0}, \mu_{0}$ and yield strength $\tau_{0}$.

The components of compressive stresses do not decrease as the fracture occurs, since the material in the fracture region remains capable of transmitting compressive forces.

In this article, to solve the problem, the method of smooth particle hydrodynamics (SPH) is used. This method includes all the necessary algorithms for accounting for the destruction of material under load and has proven itself in the study of geomechanics problems [5-9].

\section{Problem statement}

Figure 2 shows the initial configuration of the problem. The computational domain is a rectangle of $200 \times 150 \mathrm{~m}$ in size with a rectangular hole of $20 \times 20 \mathrm{~m}$, simulating a karst cavity at a depth of $50 \mathrm{~m}$. In this case, the upper boundary of region 1 is a free day surface.

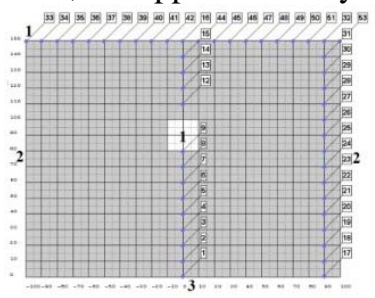

Fig.2. The initial configuration of the problem.

Boundary conditions are set on surfaces 1, 2, 3 of the computational domain, where the numbers 1 indicate free surfaces (daytime and cavity), on the surfaces 2, the conditions of complete absorption generated in the process of deformation and destruction of the load waves are realized, and on the surface 3 the condition of complete fixing is set. The points in the figure and the corresponding numbers within indicate the locations and numbers of computer sensors at which changes in the values of the massive parameters (displacements, velocities, damage $D$, etc.) are recorded.

At a certain point in time, the computational domain is loaded by gravity. As a result of this, a dynamic stress field is formed in the region, which after a certain period of time passes into a stationary one, which we will consider to be the initial one.

In order for the dynamic stage of the fracture process to be realized in the calculations, it is necessary that the strength parameters of the array are comparable with the current stresses in accordance with one or another strength criterion. It should be borne in mind that strength gradually decreases over time even with remaining constant stresses. Moreover, at some point in time, the criterion will be fulfilled and progressive failure will begin. In the future, this moment of time will be considered initial, i.e. $t=0$, for the dynamic fracture problem. 


\section{Calculation results}

As noted earlier, the formation of a funnel is, in a certain sense, the final stage of the karst process, which is gradually developing in the overlying massif. It is preceded by the formation of a cavity and cracking of the enclosing array, i.e. the formation of such a limiting state when a system of developed cracks is formed, but there is no collapse yet.

Figure 3 presents a comparison of the results of the calculation of cracking caused in a rock mass during the formation of a karst cavity with the known calculations by other numerical methods of a similar problem by other authors

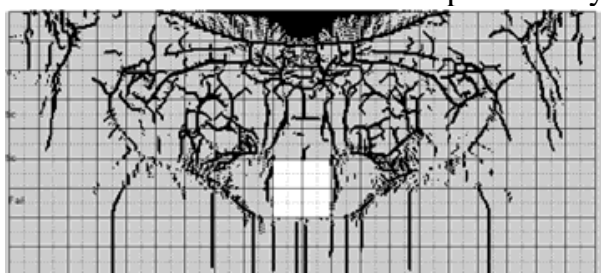

a

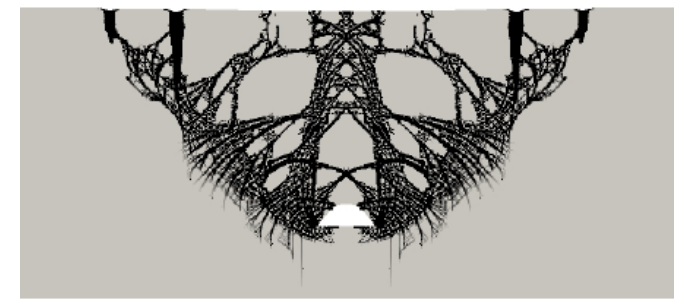

b

Fig. 3.The test calculation: simulation of this work (a) in comparison with the results of article [4].

A comparison of the results shows a qualitative agreement between the cracking patterns that characterize the process. This coincidence is confirmed by the fact that the various numerical methods used apparently adequately simulate the geomechanical phenomenon under consideration.

Figure 4 show the calculated chronograms of the development of destruction of the karst cavity and the state of the enclosing massif of rocks for various strength characteristics of the medium.

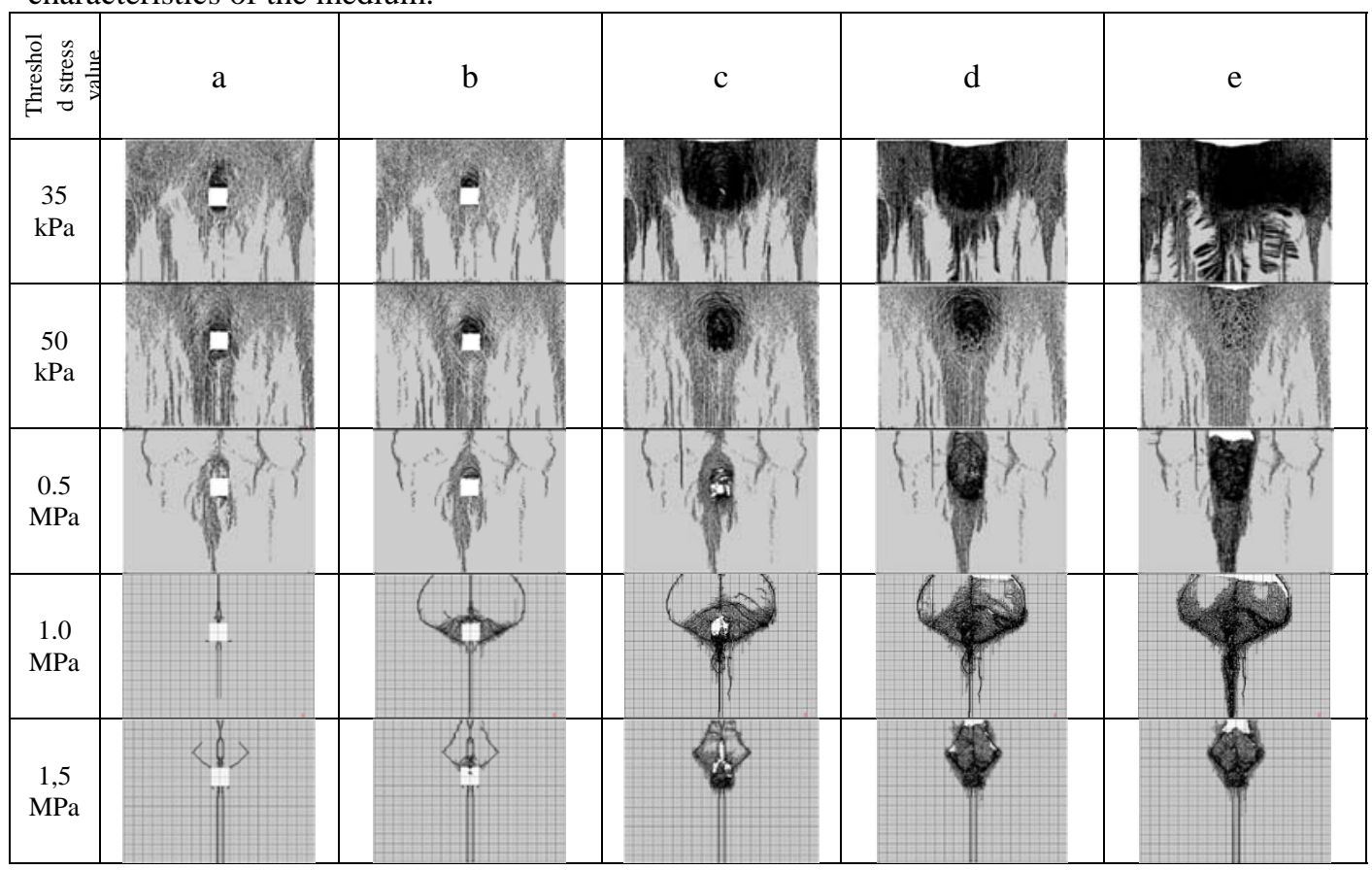

Fig.4. The calculated karst funnel formation chronogram.

During deformation of the karst cavity, a significant decrease in its vertical size and expansion in the horizontal direction occurs. Figure 4 shows the state of disturbance of the massive at some successive points in time: a - the nucleation of the main vertical crack at 
the beginning of the convergence of the cavity walls under the influence of rock pressure; $b$ - the development of subsidence of the roof of the karst cavity; c - the active stage of convergence, leading to a complete collapse of the roof; $g$ - complete collapse of the entire space of the karst cavity, which leads to subsidence of the day surface; e - the final stage of the formation of a karst funnel or failure. It should be noted that in the massif, consisting of less durable rocks, a greater loosening of the overburden occurs and a flatter depression forms in the form of a traditional settling trough. Strong rocks lead to karst funnels, such as dips with pronounced steep walls.

Note that the formation of a funnel on the surface of the earth substantially depends on the depth of the karst cavity, its horizontal dimensions, the deformation-strength properties, and the degree of loosening of the collapsing material. With strong loosening, this material can fill the developing cavity and support the roof even before the cavity comes to the surface, preventing its development. In this case, a failure does not form, but only a certain mold is formed. To one degree or another, this is reflected in the figures in the upper two rows of fig. 4. Such phenomena are observed during the development of minerals, especially salt formations, when the failure quite often comes to the earth's surface, actually destroying the mines [10].

Figure 5 shows graphs of changes in array parameters at characteristic points in response to karst formation.

\begin{tabular}{|c|c|c|}
\hline & Threshold stress value $35 \mathrm{kPa}$ & Threshold stress value $50 \mathrm{kPa}$ \\
\hline Coordinate Y & 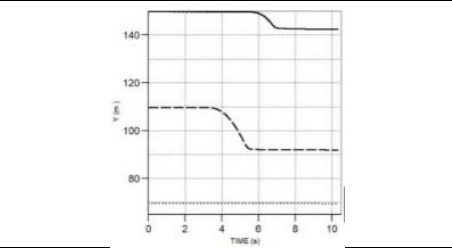 & 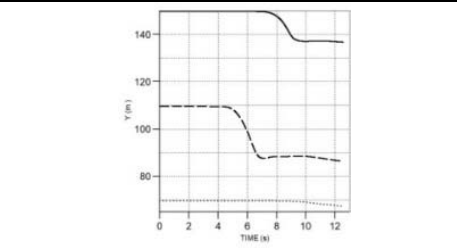 \\
\hline Vertical speed & (15: & 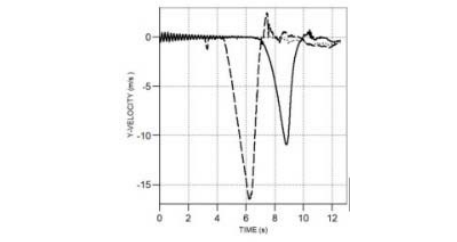 \\
\hline & Threshold stress value $1,0 \mathrm{MPa}$ & Threshold stress value $1,5 \mathrm{MPa}$ \\
\hline Coordinate Y & (1) & $\underbrace{\infty}_{0}$ \\
\hline Vertical speed & $\left.\right|_{0} ^{10}$ & 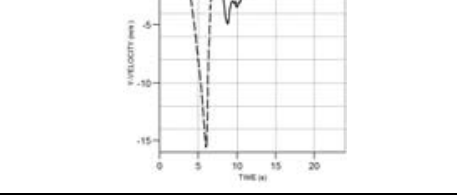 \\
\hline
\end{tabular}

Fig.5. The graphs of the change of displacement parameters of geomass - vertical displacement and speed versus of time in characteristic places.

The solid lines in Fig. 5 correspond to the parameters on the day surface at the axial point, the dotted line is the roof of the cavity, the points are the sole. On the graphs, you can notice a not entirely obvious fact that in more durable rocks the subsidence of the massive 
is more intense. The figure 6 presents the calculated seismograms of the oscillation velocity recorded in the column of seismic sensors (17-32) located in the geophysical well at a distance of $90 \mathrm{~m}$ from the karst cavity.

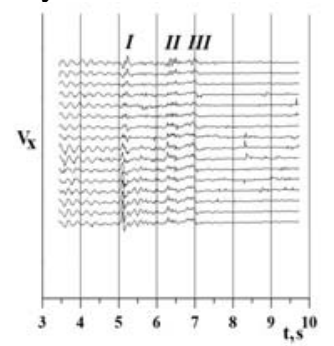

Fig.6. The calculated seismograms of vibration velocity

By the nature of the seismograms, it is possible to monitor the state of the massif subject to karst-forming activity. There are three clusters clearly traced: $\boldsymbol{I}$ - the response of the medium to the collapse of the roof of the karst cave; II - roof collapse on the sole of the cavity; III - complete co-convergence and collapse of the karst space. Thus, our observations show the explicit relation between the intensity of karst activity and the stability of the rock massive.

\section{Conclusions}

Computer simulation results show that the strength properties of rocks significantly affect the nature of the resulting fracture structures (block size, direction of cracks, width and depth of the dip), as well as the dynamics of the behavior of the geological layer above the formed karst cavity. A damaged medium with defects is subject to rapid destruction within a few seconds with the formation of subsidence of the rock either in the form of a funnel or a dip with vertical walls. The latter is characteristic of more durable covered and overlapped rocks.

The numerical analysis shows a clear relationship between the intensity of seismic destruction and the intensity of seismic radiation. Thus, the simulation can be used to develop methods for predicting the destruction of the karst cover based on computer seismic monitoring.

This work was supported by a grant 18-05-00912 from the RFBR.

\section{References}

1. N.V. Koronovsky, General geology (2006)

2. Engineering protection of territories, buildings and structures from dangerous geological processes. The main provisions. Updated edition SNiP 2202-2003 (2012)

3. G.N. Dublyanskaya, V.N. Dublyanskiy, Mapping, zoning and geotechnical assessment of karst territories (1992)

4. R. Bakeev, Yu. Stefanov, A. Myasnikov et al, AIP Conf Proc 1909 (2017)

5. V. Zakharov, V. Trofimov, I. Shipovskii, O. Malinnikova, Izvestiya Tula State University, 1 (2020)

6. I. E. Shipovskii, Scientific Bulletin of National Mining University, 1 (145) (2015)

7. V.N. Odintsev, I.E. Shipovskii, EPJ Web of Conferences 221 (2019)

8. V.N. Odintsev, I.E. Shipovskii, Journal of Mining Science, 55(4) (2019)

9. V.A. Trofimov, I.E. Shipovskii, O.N. Malinnikova, Wen-Jie Xu, AIP Conference

Proceedings, 10.1063/1.5132196 (2019) 
10. A. A. Baryakh, E. P. Rusin, S. B. Stazhevsky, A. K. Fedoseyev, G. N. Khan, Physical and Technical problems of Developing Useful Fossils, 6 (2009) 